\title{
REVIEW
}

\section{Overview of Transforming Growth Factor $\beta$ Superfamily Involvement in Glioblastoma Initiation and Progression}

\author{
André Wendindondé Nana, Pei-Ming Yang*, Hung-Yun Lin
}

\begin{abstract}
Glioblastoma, also known as glioblastoma multiforme (GBM), is the most aggressive of human brain tumors and has a stunning progression with a mean survival of one year from the date of diagnosis. High cell proliferation, angiogenesis and/or necrosis are histopathological features of this cancer, which has no efficient curative therapy. This aggressiveness is associated with particular heterogeneity of the tumor featuring multiple genetic and epigenetic alterations, but also with implications of aberrant signaling driven by growth factors. The transforming growth factor $\beta$ (TGF $\beta$ ) superfamily is a large group of structurally related proteins including TGF $\beta$ subfamily members Nodal, Activin, Lefty, bone morphogenetic proteins (BMPs) and growth and differentiation factor (GDF). It is involved in important biological functions including morphogenesis, embryonic development, adult stem cell differentiation, immune regulation, wound healing and inflammation. This superfamily is also considered to impact on cancer biology including that of GBM, with various effects depending on the member. The TGF $\beta$ subfamily, in particular, is overexpressed in some GBM types which exhibit aggressive phenotypes. This subfamily impairs anti-cancer immune responses in several ways, including immune cells inhibition and major histocompatibility (MHC) class I and II abolishment. It promotes GBM angiogenesis by inducing angiogenic factors such as vascular endothelial growth factor (VEGF), plasminogen activator inhibitor (PAI-I) and insulinlike growth factor-binding protein 7 (IGFBP7), contributes to GBM progression by inducing metalloproteinases (MMPs), "pro-neoplastic" integrins $(\alpha v \beta 3, \alpha 5 \beta 1)$ and GBM initiating cells (GICs) as well as inducing a GBM mesenchymal phenotype. Equally, Nodal promotes GICs, induces cancer metabolic switch and supports GBM cell proliferation, but is negatively regulated by Lefty. Activin promotes GBM cell proliferation while GDF yields immune-escape function. On the other hand, BMPs target GICS and induce differentiation and sensitivity to chemotherapy. This multifaceted involvement of this superfamily in GBM necessitates different strategies in anti-cancer therapy. While suppressing the TGF $\beta$ subfamily yields advantageous results, enhancing BMPs production is also beneficial.
\end{abstract}

Keywords: Glioblastoma - transforming growth factor $\beta$ (TGF $\beta$ ) superfamily - anti-TGF $\beta$ drugs

Asian Pac J Cancer Prev, 16 (16), 6813-6823

\section{Introduction}

Gliomas are heterogeneous tumors derived from glial cells or their precursors (Zong et al., 2015). They may occur in the spinal cord but arise more frequently in the brain (Ostrom et al., 2014b). Their topology can be either supratentorial (thus above the tentorium cerebelli) or infratentorial (Utsuki et al., 2012). According to the predominant cell type, gliomas can be classified into four main categories: astrocytomas arose from astrocytes, oligodendrogliomas arose from oligodendrocytes, ependymomas developed from ependymal cells, and mixed gliomas such as oligoastrocytomas (Louis et al., 2007). In addition, gliomas can be graded increasingly from 1 to 4 according to their growth rate. Grades 1 and 2 are usually well-differentiated and have better outcomes while grades 3 and 4 are undifferentiated or anaplastic and associated with a worse prognosis (Louis et al., 2007).

Glioblastoma, also known as glioblastoma multiforme
(GBM), is one of the most common and most aggressive types of malignant brain tumors, virtually without efficient therapy (Jazayeri et al., 2013; Mrugala, 2013; Ostrom et al., 2014b). It belongs to the most malignant form of astrocytoma, grade IV, along with gliosarcoma and giant cell glioblastoma (Louis et al., 2007). Histologically, GBM usually displays small areas of necrotic tissue surrounded by anaplastic cells, as well as hyperplastic blood vessels (Nakada et al., 2011). Its incidence ranges from 0.59 to 3.69 per 100,000 persons and it occurs more commonly in men than in women (Ostrom et al., 2014a).

The lack of effective therapy for GBM is due to the remarkable histological variations as different GBM subtypes can be found within the same tumor sample, to the blood-brain barrier and blood-tumor brain barrier that constitute obstacles to drug delivery and to its resistance to existing therapies, especially temozolomide, its main pharmacological approach (Sottoriva et al., 2013; Messaoudi et al., 2015; van Tellingen et al., 2015). 
Concomitantly, evidence shows an obvious implication of growth factors in some aggressive phenotypes (Rodon et al., 2014). GBM is complex and heterogeneous with regard to its cellular and molecular structure, and tumor cells interact with each other and their microenvironment via the secretion of chemokines, cytokines and growth factors in a complicated network (Bonavia et al., 2011). Nakada et al. described the overexpression of growth factors as the second main event in GBM's biology (Nakada et al., 2011). These growth factors diverted from their physiological roles, will yield aberrant signaling within the tumor cells to drive the tumor growth and metastasis, leading to a poor outcome for the patient. As a result, these growth factors and the signaling pathways they induce are continuously under intense investigation to detect possible therapeutic targets.

The TGF $\beta$ superfamily is involved in important biological functions including morphogenesis, embryonic development, adult stem cell differentiation, immune regulation, wound healing and inflammation (Santibanez et al.,2011). This superfamily is a large group of structurally related proteins that counts over 30 members in humans, divided into two branches: (1) the transforming growth factor-like (TGF-like) group with the TGF $\beta$ subfamily, Activin, Nodal and some growth and differentiation factors (GDF), and (2) the bone morphogenetic proteinlike (BMP-like) group with the BMP, GDF and antiMullerian hormone (AMH) (Weiss and Attisano, 2013). However, this protein family is implicated in the biology of various cancers including GBM, with variable effects. Some members notably the TGF $\beta$ subfamily, Activin, Nodal and GDF contribute to carcinogenesis or render tumors more aggressive, while BMP and Lefty, the antagonist of Nodal, have a tumor suppression effect. Here we provide an overview of the role of this superfamily in GBM biology, with a particular focus on the TGF $\beta$ subfamily whose implication is prominent.

\section{Classification of Glioblastoma}

The complexity of the mechanisms involved in the biology of GBM testifies to the multitude of classifications of this disease. GBM can be classified according to its mode of occurrence. In this regard, GBM can result from the progression of more benign brain tumors (secondary type) or can occur de novo (primary type). The latter is the most common form (Ohgaki and Kleihues, 2009). In addition, The World Health Organization made a classification of all brain tumors, based on the histological origin, to retrieve a clinical and prognostic significance, ranking GBM within the highest grade (grade V) (Louis et al., 2007). GBM presents a particular heterogeneity and displays tremendous genetic and epigenetic aberrations as evidenced by the results of The Cancer Genome Atlas Research Network's study (The Cancer Genome Atlas Research Network, 2008). A classification based on gene expression profile distinguishes a classical subtype, a mesenchymal subtype, a proneural subtype and a neural subtype (Verhaak et al., 2010). The classical subtype displays an abnormally high mutation rate of epidermal growth factor receptor (EGFR), no mutation of TP53 and better survival. The mesenchymal subgroup presents the most frequent mutations in the tumor suppressor NF1, frequent mutations in PTEN and TP53 and significant increase in survival after aggressive treatment. The proneural subtype contains the highest mutation in TP53, PDGFRA and IDH1 genes, and affects a younger population. The last phenotype, the neural subgroup displays several gene mutations at an average rate. This subtype occurs more often in older populations (Verhaak et al., 2010). Furthermore, a categorization of GBM based on frequent signaling pathway alteration individualizes three core pathways with key mutations: the RB pathway (CDKN2A/CDKN2C deletion, RB1 mutation, CDK4/6 amplification), the p53 pathway (CDKN2A deletion/ TP53 mutation, MDM1/4 amplification) and the receptor tyrosine kinase (RTK) pathway (Ostrom et al., 2014a). The latter pathway illuminates the particular role played by growth factors receptors mutations.

\section{Growth Factors and Glioblastoma}

In multicellular organisms, tissue homeostasis requires a constant communication between cells. Cells receive vital signals within themselves and from their immediate or distant environment that determine their fate by inducing proliferation, differentiation or death (Witsch et al., 2010). A signal-emitting cell releases a growth factor that binds to the corresponding receptor on a signal-receiving cell, to trigger a cascade of downstream events in specific pathways, leading to a cellular response, for example the expression of target genes. Because cell signaling pathways govern vital cellular processes, their disruption or hijacking is a common feature in cancer which promotes aberrant proliferation, tumor survival and ability for metastasis (Hanahan and Weinberg, 2011). Several growth factors are overexpressed in GBM, either by the tumor microenvironment or by the tumor cells. Tumor cells usually express both the growth factors and their receptors, realizing an autocrine loop (Nakada et al., 2011).

\section{Transforming Growth Factors $\beta$ (TGF $\beta$ ) Subfamily in Glioblastoma}

\section{The signaling pathways of TGF $\beta$ subfamily}

The TGF $\beta$ subfamily is a set of pleiotropic cytokines that regulate cell proliferation, differentiation and extracellular matrix production (Kaminska et al., 2013). It is composed of three isoforms (TGF $\beta 1$, TGF $\beta 2$ and TGF $\beta 3$ ), coded by three different genes (TGFBI (19q13), TGFBII (1q41) and TGFBIII (14q24)). TGF $\beta$ is synthesized initially as an inactive form called pre-proTGF $\beta$. Upon proteolysis, which proceeds in the rough endoplasmic reticulum, the pro-TGF $\beta$ is released to form a homodimer with another pro-TGF $\beta$ via disulfide bonds. Furin, a proprotein convertase, cleaves this homodimer, and drives the formation of the small latent TGF $\beta$ complex (SLC) that comprises the mature TGF $\beta$ non-covalently bound to the latency-associated protein (LAP) (Annes et al., 2003). The LAP covers the active site of TGF $\beta$, rendering it inactive until the fulfillment 
Overview of Transforming Growth Factor $\beta$ Superfamily Involvement in Glioblastoma Initiation and Progression

of certain physiological conditions. Activation of TGF $\beta$ can be processed by reactive oxygen species (ROS), mechanical stress induced by the extracellular matrix elasticity, thrombospondin-1, integrins, plasmins and several metalloproteinases (Horiguchi et al., 2012). The SLC can covalently bind to the latent transforming growth factor- $\beta$ binding protein (LTBP) to form the large latent TGF $\beta$ complex (LLC). The latter is the most common secreted form of TGF $\beta$ (Ten Dijke and Arthur, 2007). The LTBP plays a key role in the regulation of the active TGF $\beta$ availability (Rifkin, 2005). The released mature TGF $\beta$ is a dimer that binds to its specific membrane receptor to trigger an intracellular signaling pathway (Dubois et al., 1995).

TGF $\beta$ receptors (T $\beta$ Rs) belong to the serine/threonine family of membrane receptors (Massague et al., 1992). The ligand binds to a type II TGF $\beta$ receptor (T $\beta$ RII) that forms a complex with a type I TGF $\beta$ receptor (T $\beta R I)$. This leads to the phosphorylation of the downstream effectors SMAD2 or SMAD3 also called receptor-regulated SMADs
(R-SMADs). R-SMADs' phosphorylation is mediated by the SMAD anchor for receptor activation (SARA) (Tsukazaki et al., 1998). Phosphorylated R-SMADs bind to SMAD4, the common mediator SMAD (Co-SMAD). The R-SMAD/Co-SMAD complex translocates into the nucleus where it triggers the target gene expression in collaboration with other cofactors (Wrana, 1998). These SMAD cofactors vary according to the cellular context and contribute to the context-dependent feature of TGF $\beta$ 's pathway (Massague, 2012). This SMAD-dependent signaling accounts for the canonical TGF $\beta$ pathway that can be repressed by several regulators. For instance, SMAD6 and SMAD7, also termed inhibitory SMADs (I-SMADs), prevent R-SMADs' phosphorylation or their association with Co-SMAD (Miyazono, 2000). Also, SMAD ubiquitylation regulatory factors (SMURF1 and SMURF2) can target T $\beta$ RI for ubiquitination, leading to its proteosomal degradation while c-Ski and SnoN are transcriptional co-repressors that can inhibit the basal expression of TGF $\beta$ response genes via binding to the

Table 1. Current Status for the Development of anti-TGF $\beta$ Therapeutics in GBM

\begin{tabular}{|c|c|c|c|c|}
\hline Nature & Substance & Target & Mechanism & Studies \\
\hline \multirow{2}{*}{$\begin{array}{c}\text { Specific antisense } \\
\text { oligodeoxynucleotide }\end{array}$} & \multirow{2}{*}{ Trabedersen } & \multirow{2}{*}{ TGF $\beta 2$} & \multirow{2}{*}{$\begin{array}{l}\text { Represses TGF } \beta \\
\text { mRNA translation }\end{array}$} & $\begin{array}{l}\text {-In a phase I/II clinical trial, Trabedersen showed } \\
\text { prolonged survival of patients and two cases of } \\
\text { long-lasting complete tumor remissions (Hau et al., } \\
\text { 2007). }\end{array}$ \\
\hline & & & & $\begin{array}{c}\text { In a phase IIb clinical trial, } 10 \mu \mathrm{M} \text { trabedersen } \\
\text { improved GBM patient survival } 3 \text { fold at } 2 \text { and } 3 \\
\text { years compared to temozolomide, with less side } \\
\text { effects (Bogdahn et al., 2011) }\end{array}$ \\
\hline \multirow{6}{*}{ Small Molecule } & \multirow{3}{*}{ Cilengitide } & \multirow{2}{*}{$\begin{array}{l}\text { Integrins } \\
\alpha v \beta 3 \text { and } \\
\alpha v \beta 5\end{array}$} & \multirow{2}{*}{$\begin{array}{l}\text { Inhibits integrins } \\
\alpha v \beta 3, \alpha v \beta 5\end{array}$} & $\begin{array}{l}\text { In an orthotopic rat xenograft model, Cilengitide } \\
\text { potentiated radiaotherapy effects (Mikkelsen et al., } \\
\text { 2009). }\end{array}$ \\
\hline & & & & $\begin{array}{c}\text { Cilengitide monotherapy although well tolerated, } \\
\text { exhibits modest antitumor activity among recurrent } \\
\text { GBM patients in a phase II clinical trial (Reardon } \\
\text { et al., 2008). }\end{array}$ \\
\hline & & SMAD2 & $\begin{array}{c}\text { Dephosphorylates } \\
\text { SMAD2 }\end{array}$ & $\begin{array}{c}\text { Cilengitide combined to temozolomide } \\
\text { chemoradiotherapy failed to improve outcome } \\
\text { of newly diagnosed GBM patients with MGMT } \\
\text { methylation in a phase III clinical trial (Stupp et } \\
\text { al., }\end{array}$ \\
\hline & \multirow{3}{*}{ Galunisertib } & \multirow{3}{*}{$\mathrm{T} \beta \mathrm{R}$} & \multirow{3}{*}{ Inhibits $T \beta R$} & $\begin{array}{l}\text { Galunisertib is under investigation in a phase I } \\
\text { clinical trial (NCT02423343) in patients with solid } \\
\text { tumors including recurrent glioma, non-small } \\
\text { cell lung cancer, and recurrent hepatocellular } \\
\text { carcinoma. }\end{array}$ \\
\hline & & & & $\begin{array}{c}\text {-A phase Ib/IIa study combining galunisertib with } \\
\text { standard temozolomide-based radiochemotherapy } \\
\text { in patients with newly diagnosed malignant glioma } \\
\text { is ongoing under NCT01220271. }\end{array}$ \\
\hline & & & & $\begin{array}{c}\text {-An ongoing phase II clinical trial (NCT01582269) } \\
\text { is trying to assess the efficacy of galunisertib } \\
\text { alone vs lomustine or galunisertib combined with } \\
\text { lomustine in recurrent GBM. }\end{array}$ \\
\hline $\begin{array}{c}\text { Humanized } \\
\text { monoclonal antibody }\end{array}$ & Fresolimumab & $\begin{array}{l}\text { All TGF } \beta \\
\text { isoforms }\end{array}$ & $\begin{array}{l}\text { Neutralizes } \\
\text { circulating TGF } \beta \\
\text { ligands }\end{array}$ & $\begin{array}{l}\text { ·Positron emission tomography (PET) using } \\
\text { 89Zr-fresolimumab displayed a good uptake of } \\
\text { fresolimumab by high grade glioma cells, although } \\
\text { without clinical outcome (den Hollander et al., } \\
\text { 2015). }\end{array}$ \\
\hline
\end{tabular}


SMAD-response element (Kaminska et al., 2013). Always in the register of the negative regulators, BMP and Activin membrane-bound inhibitor (BAMBI) makes a stable association with the receptors of the TGF $\beta$ superfamily in order to inhibit TGF $\beta$ along with BMP (Onichtchouk et al., 1999).

Besides this canonical pathway, TGF $\beta$ can trigger Smad-independent signaling including mitogen-activated protein kinase (MAPK) pathways, Rho-like GTPase, p58, JNK-MAPK signaling pathways as well as phosphatidylinositol-3-kinase (PI3K)/AKT pathways (Mu et al., 2012).

\section{TGF $\beta$ subfamily in glioblastoma biology}

In physiological conditions and early tumor stage, TGF $\beta$ has a tumor suppressor activity via its negative growth-regulatory effect. The SMAD2/3-SMAD4 complex inhibits the mitogen c-Myc mRNA and protein expression and the inhibitor of DNA binding 1 and 3 (ID1 and ID3) (Siegel and Massague, 2003) while inducing the cyclin dependent kinase inhibitors p21WAF1/CIP1 and p15INK4b (Alexandrow and Moses, 1995). This joint action leads to the cell cycle arrest at G0/G1 phase. The induction of p15INK4b is SMAD3-dependent. Indeed, TGF $\beta$ fails to induce $\mathrm{p} 15 \mathrm{INK} 4 \mathrm{~b}$ and to inhibit astrocytic growth in Smad3-null mice compared to the wild-type (Rich et al., 1999). The induction of p21WAF1/CIP1 by the SMAD3/SMAD4 complex requires Foxo as a co-factor, which is negatively controlled by the growthpromoting PI3K pathway as well as the telencephalic development factor FoxG1 (Seoane et al., 2004). TGF $\beta$ can inhibit glioma cell growth and enhance Fas-mediated apoptosis in vivo (Ashley et al., 1998; Pan et al., 2006). However, this growth regulatory function is circumvented, moreover overturned in a tumor-promoting way in advanced cancers (Kaminska et al., 2013).

Alterations of TGF $\beta$ subfamily signaling in glioblastoma

TGF $\beta$ 's pathway can be altered in several ways. Indeed, high level of TGF $\beta$ is a common feature of this tumor, making its pathway a valuable target for drug discovery (Rodon et al., 2014). Some studies demonstrated an increase of both TGF $\beta$ receptors and ligands (Yamada et al., 1995). The SMAD proteins, downstream effectors can also be affected. However, the disturbance observed at this level is still unclear, as several studies showed contradictory results. Indeed, Zhang et al. showed a decrease of R-SMADs and the impairment of their phosphorylation and nuclear translocation upon administration of TGF $\beta 1$ to glioma cell lines. This phenomenon seemed associated with the ability of GBM to avoid the growth inhibition effect of TGF $\beta$ (Zhang et al., 2006). In contrast, other studies associated high levels of SMADs with greater malignancy and poor prognosis (Bruna et al., 2007; Rodon et al., 2014). Dieterich et al. described an increase of R-SMAD/Co-SMADs complexes in GBM endothelial cells, consistent with the angiogenesis induced by TGF $\beta$ (Dieterich et al., 2012). These apparently contradictory findings underline the complexity of the mechanism by which TGF $\beta$ pathway is hijacked in GBM.

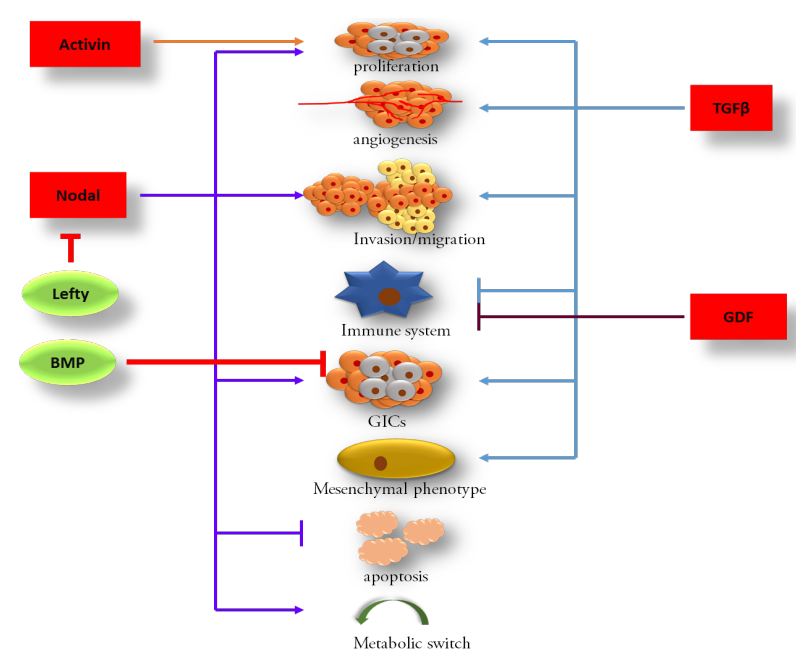

Figure 1. Complex Implication of the TGF $\beta$ Superfamily in GBM Biology. Please see the text for details. Briefly, in red, TGF $\beta$ subfamily, Nodal, Activin and GDF pomote GBM progression in several ways. In green, BMP and Lefty impair GBM by targeting glioblastoma initiating cells (GICs) and Nodal respectively

\section{Immune suppression by TGF $\beta$ subfamily}

In physiological conditions, TGF $\beta$ is an immune modulator (Travis and Sheppard, 2014). Along with the $\mathrm{T}$ regulatory (Treg) cells that it induces, TGF $\beta$ inhibits inflammation and prevents adverse effects of self-reactive T cells (Shull et al., 1992; Kulkarni et al., 1993; Wan and Flavell, 2007). TGF $\beta$ regulates both innate and adaptive immune systems (Shull et al., 1992; Yang et al., 1999). The brain is an immune-privileged organ (Bechmann and Woodroofe, 2014). This immune privilege includes, but is not only limited to the presence of the bloodbrain barrier, the lack of draining lymphatics, a dearth of professional antigen-presenting cells (APCs), such as dendritic cells (DCs), low expression levels of major histocompatibility complex (MHC) molecules; and many anti-inflammatory soluble modulators (Harris et al., 2014). Notwithstanding this immune privilege, the brain is the theater of immunological battle during the neoplastic process. Thus, high infiltration of Treg cells has a positive correlation with the grade of the tumor (Jacobs et al., 2010). GBM leverages the immune suppressor potential of Treg cells and their partner-cytokine TGF $\beta$. Indeed, TGF $\beta$ can inhibit the expression of the MHC classes I and II, in glioma cells. This allows tumor cells to invade surrounding tissues in 'stealth mode', undetected by the immune cells (Zagzag et al., 2005). Liang et al. recently demonstrated that a subset of Treg cells, the IL-17+ Tregs, utilize TGF $\beta$ to inactivate the antitumor CD8+ $\mathrm{T}$ cells (Liang et al., 2014). Ultimately, TGF $\beta$ can have an immunosuppressive effect on virtually all infiltrated immune cells including the inhibition of macrophages' activation, inhibition of T lymphocytes' and natural killer cells' maturation, differentiation or functions (Kaminska et al., 2013; Travis and Sheppard, 2014). Conversely, TGF $\beta$ 's inhibition restores the infiltration of the tumor by immune cells and enhances pro-inflammatory cytokine production in the brain (Uhl et al., 2004). 
TGF $\beta$ subfamily promotes angiogenesis

Angiogenesis is a hallmark of cancer, as proper blood supply is vital for tumor growth (Hanahan and Weinberg, 2011). In GBM, TGF $\beta$ can induce both the key angiogenic factor, vascular endothelial growth factor (VEGF) and plasminogen activator inhibitor (PAI-I) involved in the maturation of blood vessels (SanchezElsner et al., 2001; Goumans et al., 2002). Likewise, the gene expression pattern in GBM new vessels testifies to the importance of TGF $\beta$-driven angiogenesis. Dieterich et al. characterized the transcriptome profile in GBM new vessels and identified 95 genes differentially expressed. An enrichment in SMADs' target genes was observed, including COL1A2 and COL3A1 involved in extracellular matrix (ECM) organization, and FN1 implicated in wound healing (Dieterich et al., 2012). Furthermore, insulin-like growth factor-binding protein 7 (IGFBP7) is an insulin growth factor carrier that is strongly expressed in tumor endothelial cells and vascular basement membrane. IGFBP7 is considered as a biomarker of GBM tumor vessel and can be targeted by a specific antibody for molecular imaging (Iqbal et al., 2010). IGFBP7 has proangiogenic properties and the IGFBP family elsewhere is incriminated in oncogenesis (Baxter, 2014). IGFBP7 is described to induce the formation of an extensive network of capillary-like tubes of human brain endothelial cells, consecutive to TGF $\beta$ administration. This upregulation of IGFBP7 by TGF $\beta$ requires ALK/SMAD2 pathway's activation (Pen et al., 2008). This in vitro finding suggests that TGF $\beta$ may utilize IGBP7 which is abundantly expressed in GBM vessels, to promote tumor vessels formation.

\section{TGF $\beta$ subfamily promotes tumor invasion}

GBM is a highly invasive tumor (Mrugala, 2013). Invasion of tumor cells into the surrounding tissue is a complex process that requires modifications in cell-cell contacts, cell-matrix contacts and degradation of the local ECM by proteases such as membrane metalloproteinases (MMPs) (Luwor et al., 2008). Integrins are cell adhesion molecules that are also membrane receptors (Bergh et al., 2005). They mediate interactions between cells and ECM, facilitate ECM-dependent organization of the cytoskeleton and activate intracellular signaling required for the regulation of cell adhesion and migration (D'Abaco and Kaye, 2007). Integrin's role in tumor progression has been well established. Tumor cells commonly reorganize integrins at their surface by repressing "anti-neoplastic" integrins, such as $\alpha 2 \beta 1$ and $\alpha 3 \beta 1$, while they promote the "pro-neoplastic" ones, including $\alpha \mathrm{v} \beta 3, \alpha \mathrm{v} \beta 6, \alpha 5 \beta 1$ or $\alpha 6 \beta 4$ (Guo and Giancotti, 2004; Schaffner et al., 2013). The integrins can activate TGF $\beta$ by inducing the release of the LAP (Horiguchi et al., 2012). Reciprocally, in several cancers, TGF $\beta$ promotes tumor progression and metastasis by increasing integrin's expression (Heino and Massague, 1989; Fong et al., 2009). Indeed, on glioma cells, TGF- $\beta$ enhances integrin $\alpha v \beta 3$ 's expression to promote tumor migration; this effect is inhibited by echistatin, the Arg-Gly-Asp (RGD) peptide antagonist of $\alpha v \beta 3$ integrin (Platten et al., 2000). Also, GBM cells expressing high level of $\alpha 5 \beta 1$ integrin display aggressive phenotypes and the scaffolding protein Caveolin-1 has been described to negatively regulate this process (Martin et al., 2009). Caveolin-1 abrogates $\alpha 5 \beta 1$ integrin expression in GBM, by repressing the canonical TGF $\beta$ pathway which is the $\alpha 5 \beta 1$ inducer (Cosset et al., 2012). Furthermore, the neural cell adhesion molecule L1 that belongs to the immunoglobulin superfamily has also been described to induce glioma invasion (Izumoto et al., 1996). TGF $\beta$ has been described to enhance the expression of L1 to promote GBM invasiveness (Luwor et al., 2008). Moreover, several studies illuminated the role of TGF $\beta$ in the induction of MMPs. TGF $\beta$ can create an imbalance by upregulating MMPs and repressing tissue inhibitor of metalloproteinase (TIMP) (Rao et al., 1993; Nakagawa et al., 1994; Wick et al., 2001; Friese et al., 2004). In this regard, the microenvironment plays an important role. Indeed, tumor-associated macrophages can secrete TGF $\beta$ that in turn induces the GBM stem cells to release MPPs (Ye et al., 2012).

In sum, TGF $\beta$ promotes angiogenesis and provides proper blood supply to neoplastic cells. It represses antitumor immune response, induces MMPs and represses TIMP, and finally, induces "pro-neoplastic" integrin's expression. By doing so, TGF $\beta$ masterfully orchestrates complex events that ultimately buttress tumor invasion of the normal brain parenchyma.

\section{TGF $\beta$ subfamily induces epithelial-mesenchymal transition (EMT) and cancer stemness}

Epithelial-mesenchymal transition (EMT) is the early step in the cancer invasion and metastasis process, where epithelial cells lose their polarity to acquire a mesenchymal phenotype (Scheel and Weinberg, 2012). This phenomenon is driven by well-known transcription factors namely Snail, Twist and zinc-finger E-box binding homeobox 1 (ZEB1) (Sánchez-Tilló et al., 2012). In the particular case of GBM, TGF $\beta$, via SMAD signaling, can induce ZEB1 which in turn drives a mesenchymal transdifferentiation with enhanced tumor migration (Joseph et al., 2014).

GBM, like many other cancers, exhibits a subset of cells that present self-renewal and differentiation ability, justifying the concept of cancer stem cells (Singh et al., 2004). The GBM stem cells or GBM initiating cells (GICs) entail chemo-resistance and radio-resistance and are the subjects of multiple therapeutic investigations with mixed outcomes as yet (Cho et al., 2013). These cells express higher level of TGF $\beta$ compared to differentiated tumor cells (Qiu et al., 2011). TGF $\beta$ induces several factors that in turn promote tumor cell stemness. For instance, TGF $\beta$ has been described to promote the formation of these stem cells via induction of leukemia inhibitory factor (LIF) and the JAK-STAT pathway (Penuelas et al., 2009). LIF, elsewhere has been described to promote the self-renewal capacity of mouse embryonic stem cells via activation of STAT3 and the CP2 family transcription factor Tfcp211 (Ye et al., 2013). Moreover, TGF $\beta$ induces Sox 2 expression in an autocrine way, to maintain stemness of GICs (Ikushima et al., 2009). Also, in normal cells, TGF $\beta$ 's pathway inhibits ID1 and ID3 to drive cell cycle arrest at G0/G1(Siegel and Massague, 2003). However, in 
cancer cells including GBM, ID1 can be induced by TGF $\beta$ (Goumans et al., 2002) to promote tumor progression. Conversely, TGF $\beta$ 's inhibition in GICs expressing high levels of ID1 decreased these cells' population concomitantly as it reduced ID1 and ID3 expression (Anido et al., 2010). TGF $\beta$-mediated actions on GICs may depend on the GBM subtype. Indeed, GICs of the proneural subtype, while less responsive to TGF $\beta$ 's action, secrete TGF $\beta$ and utilize TGF $\beta$ immunosuppressive ability to reduce the infiltration of immune cells. On the other hand, GICs of the mesenchymal subtype seem more sensitive to TGF $\beta$ 's anti or pro-proliferative effect but present more infiltrated immune cells (Beier et al., 2012). The tumor microenvironment is a well-known contributor to cancer progression. GBM's tumor associated microglia/ macrophages secrete TGF $\beta 1$ that binds to overexpressed T $\beta$ RII at GICs surface, triggering the latter's MMP9 release to promote tumor invasion (Ye et al., 2012).

\section{Other Members of TGF $\beta$ Superfamily implicated in Glioblastoma biology}

Even if TGF $\beta$ subfamily overwhelmingly drives GBM progression and is the subject of research focus, the other growth factors of the superfamily nevertheless play a role in this tumor's biology. These growth factors, mainly BMPs, Activins, Nodal and GDFs regulate early vertebrate development and adult tissue maintenance but are also incriminated in some tumors development and metastasis (Wakefield and Hill, 2013). However, their effects on GBM evolution are antagonistic.

\section{Bone morphogenetic proteins (BMPs)}

In the BMPs canonical signaling, like in TGF $\beta$ pathway, R-SMADs (SMAD1, SMAD5 and SMAD8) are phosphorylated upon activation of BMP receptor of the serine/threonine family of membrane receptors. Activated R-SMADs form a complex with Co-SMAD (SMAD4) and the activated R-SMAD/Co-SMAD complex then translocates into the nucleus to activate BMPs transcriptional targets (Wrana, 2013). BMPs have diverse functions in embryonic brain development. For instance, BMPs trigger neuronal differentiation of precursors in the neocortical ventricular zone and promote astroglial lineage commitment of mammalian subventricular zone progenitor cells (Gross et al., 1996; Li et al., 1998). Neurogenesis in the adult brain is restricted to specific neurogenic regions located in the hippocampus, the subventricular zone, the striatum and the olfactory bulb (Ernst and Frisen, 2015). Along with their negative regulator Noggin, BMPs play an important regulatory role in neural stem cells niche (Lim et al., 2000).

Apart from their multiple roles in physiological processes in the brain, BMPs are involved in cancer biology. However, in contrast to TGF $\beta$, BMPs have a tumor suppressor activity in GBM. Indeed, BMPs, especially BMP4, reduce GBM CD133+ cancer stem cell proliferation, induce their differentiation, and abolish their tumor formation and subsequent mortality in xenograft model (Piccirillo and Vescovi, 2006). This antitumor effect is mediated by the BMP/SMAD/SNAIL pathway
(Savary et al., 2013).

Moreover, the resistance to temozolomide induced by GICs is suppressed by BMPs. High expression of O6-methylguanine-DNA-methyltransferase (MGMT) and hypoxia-inducible factor- $1 \alpha(\mathrm{HIF}-1 \alpha)$ is a common feature of GICs that generates resistance to temozolomide. BMP2 downregulates both MGMT and HIF-1 $\alpha$ in GICs, leading to their differentiation and improving their sensitivity to temozolomide (Persano et al., 2012).

Because of their antitumor properties especially directed toward the GICs, BMPs are seen as attractive therapeutic tools that can allow to "cut the evil at its root". For this purpose, nanocomplex encapsulation technique may be a good approach for BMP delivery to the target site (Reguera-Nuñez et al., 2014). However, cancer cells are not short of tricks. Indeed, certain tumor cells circumvent this phenomenon by producing BMPs antagonists such as Gremlin1 that inhibits the BMP's canonical pathway and potentiate the tumor's growth (Yan et al., 2014).

\section{Nodal}

In contrast to BMPs, Nodal promotes GBM cell growth. Nodal is essential for the induction of body axis during embryogenesis. Nodal triggers SMADs signaling pathway but also induces non-SMADs signaling such as extracellular-signal-regulated kinase (ERK) signal transduction pathway. Nodal, which is normally expressed in embryonic and reproductive cell types, is more and more described in tumor cells where it yields aggressive cancer phenotypes (Quail et al., 2013). GBM cells transfected with plasmid encoding Nodal show high proliferation rate upon activation of the Nodal receptors ALK4, ALK7 and SMAD3 phosphorylation (De Silva et al., 2012). Nodal stimulates tumor invasion by upregulating MMP2 and may also promote GICs via induction of LIF and Cripto-1 (Lee et al., 2010). In sum, Nodal promotes GBM cancer cells proliferation, invasion and may also support the stemness of GICs. Interestingly, TGF $\beta$ can enhance Nodal expression via ERK1/2 activation, to promote tumor proliferation and invasion, but inhibit apoptosis (Sun et al., 2014b).

Energy metabolism reprogramming is a well-known trick that cancer cells develop and constitute a hallmark of cancer (Hanahan and Weinberg, 2011). Albeit uneconomic energy wise, cancer cells commonly switch from the pyruvate pathway to glycolysis by upregulating HIF $1 \alpha$ and HIF $2 \alpha$, leading to higher glucose uptake, low oxygen consumption and low mitochondrial metabolism. This process renders them more resistant to the hypoxic condition common in cancer. In GBM, Nodal triggers this metabolic switch by upregulating HIF1 $\alpha$ (Lai et al., 2013).

However, Nodal tumor promotion is negatively regulated by Lefty, another member of the TGF $\beta$ superfamily. Indeed, Lefty inhibits Nodal expression, and impairs Nodal-induced expression of SMADs and activation of ERK1/2. It further inhibits cells proliferation and tumor invasion and enhances apoptosis (Sun et al., 2014a).

\section{Activins}

Activins are ubiquitous growth factors that regulate 
functions as diverse as gonadal function, hormonal homeostasis, growth and differentiation of musculoskeletal tissues, brain functions, proliferation and differentiation of embryonic stem cells and also cancer growth and metastasis (Tsuchida et al., 2009). Activins bind to their serine-threonine kinase receptor to trigger SMAD signaling pathway and non-SMAD pathways including MAPK, PI3K/AKT and Wnt/ $\beta$-catenin signaling (Tsuchida et al., 2009). Activin A induced human GBM cells U87 proliferation via SMAD2/3 activation; this effect is negatively regulated by Follistatin that is an Activinbinding protein (Zhang et al., 2010).

\section{Growth and differentiation factor (GDF)}

GDF has been described to induce GBM progression. Indeed, GDF is found overexpressed in high-grade glioma cells where it yields an immune-escape function and correlates with poor patient survival. Likewise, GBM cells deprived of GDF become more vulnerable to natural killer cells (Roth et al., 2010). However, other sources suggest that GDF may be secreted by the tumor-infiltrating macrophages instead of the GBM tumor cells (Shnaper et al., 2009).

\section{Crosstalk between TGF $\beta$ and Other Growth Factors in Glioblastoma}

In GBM, as in cancer in general, signaling pathways usually overlap, creating cross-talks that eventually participate in the cancer's trick to escape from endogenous repressions as well as exogenous threats represented by anti-cancer therapy. Thus, several pathways taken individually can lead to a same outcome, testifying to the redundancy of such a phenomenon. Considering GBM's angiogenesis, TGF $\beta$, PDGF-B and mostly HIF-1 can induce VEGF, the main contributor in this process. TGF $\beta$ can cooperate with both PDGF and EGF. Nonetheless, the interaction between TGF $\beta$, PDGF and EGF is somewhat controversial. Indeed, some studies demonstrated that TGF $\beta$ can induce PDGF and EGF to promote GBM (Helseth et al., 1988; Bruna et al., 2007) while other evidence showed an inhibition of the same transcription factors by the TGF $\beta$ in some gliomas (Vergeli et al., 1995; Held-Feindt et al., 2003). The pleiotropic nature of TGF $\beta$, whose effect is regulated by SMAD contextual partners may be a rational explanation for these differences.

\section{Therapeutic Perspectives}

In GBM, as in other cancers, the role of TGF $\beta$ subfamily is ambivalent. Initially protective, it switches later to promote the tumor progression. Differentiating when it protects from when it harms is of crucial importance. Shen et al. demonstrated that the epigenetic silencing of the tumor suppressor NDRG2 leads to TGF $\beta$ 's about-face in colon cancer while $\mathrm{Xu}$ et al. identified the adaptor protein $14-3-3 \zeta$ as the agent responsible for TGF $\beta$ 's wrong turn in breast cancer (Shen et al., 2014; $\mathrm{Xu}$ et al., 2015). The discovery of the causative agents of the same mechanism in GBM will be of unquestionable therapeutic importance.
Yet, several anti-TGF $\beta$ drugs have been developed even if the outcomes remain unsatisfactory (Table 1). Trabedersen, an antisense oligonucleotide of TGF $\beta$, has been developed and successfully tested in phase I/II clinical trials (Hau et al., 2007). Trabedersen is a first-in-class gene silencing antisense compound (phosphorothioate oligodeoxynucleotide) designed to selectively downregulate the production of TGF $\beta 2$ at the translational level. A phase III clinical trial initiated in September 2008 was stopped in March 2012 owing to neurosurgical procedures and first-line standard of care for patients that made impossible to recruit the projected number of patients (Akhurst and Hata, 2012).

Additionally, inhibitors of integrin receptors have been found therapeutically relevant in GBM. Integrins are involved in TGF $\beta$ activation by mechanical pulling of the large latent complex (Horiguchi et al., 2012). When treated with integrin inhibitors, glioma cells undergo atypical cell death in combination of anoikis, apoptosis and necrosis, which can be rescued by TGF $\beta$ treatment (Silginer et al., 2014). Cilengitide is an antiangiogenic small molecule, an inhibitor of $\alpha v \beta 3$ and $\alpha v \beta 5$ integrin, that prevents TGF $\beta$ 's tumor promotion in glioma cells by decreasing TGF $\beta$ level and dephosphorylating SMAD2 (Roth et al., 2013). After promising results in animal models (Mikkelsen et al., 2009), this molecule has been translated to clinical trials with as-yet unsatisfactory results (Stupp et al.; Reardon et al., 2008; Eisele et al., 2014)

Blocking the abnormal signal at its origin by inhibiting TGF $\beta$ receptor is another strategy to prevent its harmful effect. Thus, small molecules have been employed against T $\beta$ Rs. However, these small molecules are subject to nonspecific binding with resultant "off-target" effects. Galunisertib (LY2157299) is an example tested in phase Ib/IIa clinical trials (Akhurst and Hata, 2012; Katz et al., 2013).

In contrast, antibodies offer a more targeted approach in anti-TGF $\beta$ based therapy. Fresolimumab is a human monoclonal antibody that can neutralize all TGF $\beta$ 's isoforms with high-affinity in several cancer cell lines. This antitumor property led to its clinical translation in several cancers including GBM, malignant melanoma and renal cell carcinoma attesting to its safety and potential therapeutic effect (Morris et al., 2014). Nonetheless, fresolimumab can induce skin lesions, which require caution (Lacouture et al., 2015). Efficient drug delivery is an important aspect of cancer treatment, especially in the brain, which is protected by the blood-brain barrier. Recently, den Hollander et al. demonstrated a satisfactory uptake of fresolimumab by high-grade glioma cells, yet with no clinical outcome. (den Hollander et al., 2015). Further investigation should provide more insight as to the efficacy of this drug in GBM.

\section{Conclusion}

In sum, the involvement of the TGF $\beta$ superfamily in GBM biology is multidimensional. The TGF $\beta$ subfamily orchestrates complex events involving cancer cell proliferation and migration, immune suppression, angiogenesis, mesenchymal phenotype induction and 
GICs promotion. The conjunction of these pro-tumoral functions underlies the aggressive phenotypes exhibited by tumors with high level of TGF $\beta$ and justifies the need to target this growth factor for drug development. In the same way, Nodal, Activin and GDF promote GBM progression, whereas BMPs and Lefty, by targeting GICs and nodal respectively, impair GBM growth.

\section{Acknowledgements}

We would like to thank Jorie Larson MPH for her contribution in proofreading this paper. This work was supported by Taipei Medical University (TMU104AE2-I02-4) and Comprehensive Cancer Center of Taipei Medical University (MOHW104-TDU-B-212-124-001) funded by Health and welfare surcharge of tobacco products.

\section{References}

Akhurst RJ, Hata A (2012). Targeting the TGFbeta signalling pathway in disease. Nat Rev Drug Discov, 11, 790-811.

Alexandrow MG, Moses HL (1995). Transforming growth factor beta and cell cycle regulation. Cancer Res, 55, 1452-7.

Anido J, Saez-Borderias A, Gonzalez-Junca A, et al (2010). TGFbeta Receptor Inhibitors Target the CD44(high)/Id1(high) Glioma-Initiating Cell Population in Human Glioblastoma. Cancer Cell, 18, 655-68.

Annes JP, Munger JS, Rifkin DB (2003). Making sense of latent TGFbeta activation. J Cell Sci, 116, 217-24.

Ashley DM, Kong FM, Bigner DD, et al (1998). Endogenous expression of transforming growth factor beta1 inhibits growth and tumorigenicity and enhances Fas-mediated apoptosis in a murine high-grade glioma model. Cancer Res, 58, 302-9.

Baxter RC (2014). IGF binding proteins in cancer: mechanistic and clinical insights. Nat Rev Cancer, 14, 329-41.

Bechmann I, Woodroofe N (2014). Immune Privilege of the Brain. In 'Neuroinflammation and CNS Disorders', Eds John Wiley \& Sons, Ltd, 1-8

Beier CP, Kumar P, Meyer K, et al (2012). The cancer stem cell subtype determines immune infiltration of glioblastoma. Stem Cells Dev, 21, 2753-61.

Bergh JJ, Lin HY, Lansing L, et al (2005). Integrin alphaVbeta3 contains a cell surface receptor site for thyroid hormone that is linked to activation of mitogen-activated protein kinase and induction of angiogenesis. Endocrinol, 146, 2864-71.

Bonavia R, Inda MM, Cavenee WK, et al (2011). Heterogeneity maintenance in glioblastoma: a social network. Cancer Res, 71, 4055-60.

Bruna A, Darken RS, Rojo F, et al (2007). High TGFbeta-Smad activity confers poor prognosis in glioma patients and promotes cell proliferation depending on the methylation of the PDGF-B gene. Cancer Cell, 11, 147-60.

Cho DY, Lin SZ, Yang WK, et al (2013). Targeting cancer stem cells for treatment of glioblastoma multiforme. Cell Transplant, 22, 731-9.

Cosset EC, Godet J, Entz-Werle N, et al (2012). Involvement of the TGF $\beta$ pathway in the regulation of $\alpha 5 \beta 1$ integrins by caveolin-1 in human glioblastoma. Int J Cancer, 131, 601-11.

D'Abaco GM, Kaye AH (2007). Integrins: molecular determinants of glioma invasion. J Clin Neurosci, 14, 1041-8.

De Silva T, Ye G, Liang YY, et al (2012). Nodal promotes glioblastoma cell growth. Front Endocrinol (Lausanne),
3, 59 .

den Hollander MW, Bensch F, Glaudemans AW, et al (2015). TGF-beta antibody uptake in recurrent high grade glioma imaged with $89 \mathrm{Zr}$-fresolimumab PET. J Nucl Med.

Dieterich LC, Mellberg S, Langenkamp E, et al (2012). Transcriptional profiling of human glioblastoma vessels indicates a key role of VEGF-A and TGF 32 in vascular abnormalization. J Pathol, 228, 378-90.

Dubois CM, Laprise MH, Blanchette F, et al (1995). Processing of transforming growth factor beta 1 precursor by human furin convertase. J Biol Chem, 270, 10618-24.

Eisele G, Wick A, Eisele AC, et al (2014). Cilengitide treatment of newly diagnosed glioblastoma patients does not alter patterns of progression. $J$ Neurooncol, 117, 141-5.

Ernst A, Frisen J (2015). Adult Neurogenesis in HumansCommon and Unique Traits in Mammals. PLoS Biol, 13, 1002045.

Fong YC, Hsu SF, Wu CL, et al (2009). Transforming growth factor-beta1 increases cell migration and betal integrin up-regulation in human lung cancer cells. Lung Cancer, 64, 13-21.

Friese MA, Wischhusen J, Wick W, et al (2004). RNA interference targeting transforming growth factor-beta enhances NKG2Dmediated antiglioma immune response, inhibits glioma cell migration and invasiveness, and abrogates tumorigenicity in vivo. Cancer Res, 64, 7596-603.

Goumans MJ, Valdimarsdottir G, Itoh S, et al (2002). Balancing the activation state of the endothelium via two distinct TGFbeta type I receptors. Embo J, 21, 1743-53.

Gross RE, Mehler MF, Mabie PC, et al (1996). Bone morphogenetic proteins promote astroglial lineage commitment by mammalian subventricular zone progenitor cells. Neuron, 17, 595-606.

Guo W, Giancotti FG (2004). Integrin signalling during tumour progression. Nat Rev Mol Cell Biol, 5, 816-26.

Hanahan D, Weinberg RA (2011). Hallmarks of cancer: the next generation. Cell, 144, 646-74.

Harris MG, Hulseberg P, Ling C, et al (2014). Immune privilege of the CNS is not the consequence of limited antigen sampling. Sci Rep, 4.

Hau P, Jachimczak P, Schlingensiepen R, et al (2007). Inhibition of TGF-beta2 with AP 12009 in recurrent malignant gliomas: from preclinical to phase I/II studies. Oligonucleotides, 17, 201-12.

Heino J, Massague J (1989). Transforming growth factor-beta switches the pattern of integrins expressed in MG-63 human osteosarcoma cells and causes a selective loss of cell adhesion to laminin. J Biol Chem, 264, 21806-11.

Held-Feindt J, Lutjohann B, Ungefroren H, et al (2003). Interaction of transforming growth factor-beta (TGF-beta) and epidermal growth factor (EGF) in human glioma cells. $J$ Neurooncol, 63, 117-27.

Helseth E, Dalen A, Unsgaard G, et al (1988). Type beta transforming growth factor and epidermal growth factor suppress the plasminogen activator activity in a human glioblastoma cell line. J Neurooncol, 6, 277-83.

Horiguchi M, Ota M, Rifkin DB (2012). Matrix control of transforming growth factor- $\beta$ function. $J$ Biochem, 152, 321-9.

Ikushima H, Todo T, Ino Y, et al (2009). Autocrine TGF- $\beta$ signaling maintains tumorigenicity of glioma-initiating cells through sry-related HMG-Box factors. Cell Stem Cell, 5, 504-14.

Iqbal U, Albaghdadi H, Luo Y, et al (2010). Molecular imaging of glioblastoma multiforme using anti-insulin-like growth factor-binding protein-7 single-domain antibodies. British J Cancer, 103, 1606-16. 
Overview of Transforming Growth Factor $\beta$ Superfamily Involvement in Glioblastoma Initiation and Progression

Izumoto S, Ohnishi T, Arita N, et al (1996). Gene expression of neural cell adhesion molecule L1 in malignant gliomas and biological significance of L1 in glioma invasion. Cancer Res, 56, 1440-4.

Jacobs JF, Idema AJ, Bol KF, et al (2010). Prognostic significance and mechanism of Treg infiltration in human brain tumors. J Neuroimmunol, 225, 195-9.

Jazayeri SB, Rahimi-Movaghar V, Shokraneh F, et al (2013). Epidemiology of primary CNS tumors in Iran: a systematic review. Asian Pac J Cancer Prev, 14, 3979-85.

Joseph JV, Conroy S, Tomar T, et al (2014). TGF-[beta] is an inducer of ZEB 1-dependent mesenchymal transdifferentiation in glioblastoma that is associated with tumor invasion. Cell Death Dis, 5, 1443.

Kaminska B, Kocyk M, Kijewska M (2013). TGF beta signaling and its role in glioma pathogenesis. Adv Exp Med Biol, 986, 171-87.

Katz LH, Li Y, Chen JS, et al (2013). Targeting TGF-beta signaling in cancer. Expert Opin Ther Targets, 17, 743-60.

Kulkarni AB, Huh CG, Becker D, et al (1993). Transforming growth factor beta 1 null mutation in mice causes excessive inflammatory response and early death. Proc Natl Acad Sci U S A, 90, 770-4.

Lacouture M, Morris J, Lawrence D, et al (2015). Cutaneous keratoacanthomas/squamous cell carcinomas associated with neutralization of transforming growth factor $\beta$ by the monoclonal antibody fresolimumab (GC1008). Cancer Immunol Immunother, 64, 437-46.

Lai J-H, Jan H-J, Liu L-W, et al (2013). Nodal regulates energy metabolism in glioma cells by inducing expression of hypoxia-inducible factor $1 \alpha$. Neuro-Oncol, 15, 1330-41.

Lee CC, Jan HJ, Lai JH, et al (2010). Nodal promotes growth and invasion in human gliomas. Oncogene, 29, 3110-23.

Li W, Cogswell CA, LoTurco JJ (1998). Neuronal differentiation of precursors in the neocortical ventricular zone is triggered by BMP. J Neurosci, 18, 8853-62.

Liang H, Yi L, Wang X, et al (2014). Interleukin-17 facilitates the immune suppressor capacity of high-grade gliomaderived CD4 (+) CD25 (+) Foxp3 (+) T cells via releasing transforming growth factor beta. Scand J Immunol, 80, 144-50.

Lim DA, Tramontin AD, Trevejo JM, et al (2000). Noggin antagonizes BMP signaling to create a niche for adult neurogenesis. Neuron, 28, 713-26.

Louis DN, Ohgaki H, Wiestler OD, et al (2007). The 2007 WHO classification of tumours of the central nervous system. Acta Neuropathol, 114.

97-109.

Luwor RB, Kaye AH, Zhu HJ (2008). Transforming growth factor-beta (TGF-beta) and brain tumours. J Clin Neurosci, 15, 845-55

Martin S, Cosset EC, Terrand J, et al (2009). Caveolin-1 regulates glioblastoma aggressiveness through the control of alpha(5) beta(1) integrin expression and modulates glioblastoma responsiveness to SJ749, an alpha(5)beta(1) integrin antagonist. Biochim Biophys Acta, 1793, 354-67.

Massague J (2012). TGFbeta signalling in context. Nat Rev Mol Cell Biol, 13, 616-30.

Massague J, Andres J, Attisano L, et al (1992). TGF-beta receptors. Mol Reprod Dev, 32, 99-104.

Messaoudi K, Clavreul A, Lagarce F (2015). Toward an effective strategy in glioblastoma treatment. Part I: resistance mechanisms and strategies to overcome resistance of glioblastoma to temozolomide. Drug Discovery Today.

Mikkelsen T, Brodie C, Finniss S, et al (2009). Radiation sensitization of glioblastoma by cilengitide has unanticipated schedule-dependency. Int J Cancer, 124, 2719-27.
Miyazono K (2000). Positive and negative regulation of TGFbeta signaling. J Cell Sci, 113, 1101-9.

Morris JC, Tan AR, Olencki TE, et al (2014). Phase i study of gc1008 (fresolimumab): a human anti-transforming growth factor-beta $(\operatorname{tgf} \beta)$ monoclonal antibody in patients with advanced malignant melanoma or renal cell carcinoma. PLOS ONE, 9, 90353.

Mrugala MM (2013). Advances and challenges in the treatment of glioblastoma: a clinician's perspective. Discov Med, 15, 221-30.

Mu Y, Gudey S, Landstrom M (2012). Non-Smad signaling pathways. Cell Tissue Res, 347, 11-20.

Nakada M, Kita D, Watanabe T, et al (2011). Aberrant signaling pathways in glioma. Cancers (Basel), 3, 3242-78.

Nakagawa T, Kubota T, Kabuto M, et al (1994). Production of matrix metalloproteinases and tissue inhibitor of metalloproteinases-1 by human brain tumors. J Neurosurg, 81, 69-77.

Ohgaki H, Kleihues P (2009). Genetic alterations and signaling pathways in the evolution of gliomas. Cancer Sci, 100, 2235-41.

Onichtchouk D, Chen YG, Dosch R, et al (1999). Silencing of TGF-beta signalling by the pseudoreceptor BAMBI. Nature, 401, 480-5.

Ostrom QT, Bauchet L, Davis FG, et al (2014a). The epidemiology of glioma in adults: a "state of the science" review. Neuro Oncol, 16, 896-913.

Ostrom QT, Gittleman H, Liao P, et al (2014b). CBTRUS statistical report: primary brain and central nervous system tumors diagnosed in the United States in 2007-2011. NeuroOncol, 16, 1-63.

Pan JJ, Chang WJ, Barone TA, et al (2006). Increased expression of TGF-beta1 reduces tumor growth of human U-87 Glioblastoma Cells in vivo. Cancer Immunology, Immunotherapy, 55, 918-27.

Pen A, Moreno MJ, Durocher Y, et al (2008). Glioblastomasecreted factors induce IGFBP7 and angiogenesis by modulating Smad-2-dependent TGF-[beta] signaling. Oncogene, 27, 6834-44.

Penuelas S, Anido J, Prieto-Sanchez RM, et al (2009). TGFbeta increases glioma-initiating cell self-renewal through the induction of LIF in human glioblastoma. Cancer Cell, 15, 315-27.

Persano L, Pistollato F, Rampazzo E, et al (2012). BMP2 sensitizes glioblastoma stem-like cells to Temozolomide by affecting HIF- $1 \alpha$ stability and MGMT expression. Cell Death Disease, 3, 412.

Piccirillo SG, Vescovi AL (2006). Bone morphogenetic proteins regulate tumorigenicity in human glioblastoma stem cells. Ernst Schering Found Symp Proc, 59-81.

Platten M, Wick W, Wild-Bode C, et al (2000). Transforming growth factors beta(1) (TGF-beta(1)) and TGF-beta(2) promote glioma cell migration via Up-regulation of alpha(V) beta(3) integrin expression. Biochem Biophys Res Commun, 268, 607-11.

Qiu B, Zhang D, Wang C, et al (2011). IL-10 and TGF- 32 are overexpressed in tumor spheres cultured from human gliomas. Molecular Biology Reports, 38, 3585-91.

Quail DF, Siegers GM, Jewer M, et al (2013). Nodal signalling in embryogenesis and tumourigenesis. Int J Biochem Cell Biol, 45, 885-98.

Rao JS, Steck PA, Mohanam S, et al (1993). Elevated levels of M(r) 92,000 type IV collagenase in human brain tumors. Cancer Res, 53, 2208-11.

Reardon DA, Fink KL, Mikkelsen T, et al (2008). Randomized phase II study of cilengitide, an integrin-targeting arginineglycine-aspartic acid peptide, in recurrent glioblastoma 
multiforme. J Clin Oncol, 26, 5610-7.

Reguera-Nuñez E, Roca C, Hardy E, et al (2014). Implantable controlled release devices for BMP-7 delivery and suppression of glioblastoma initiating cells. Biomaterials, 35, 2859-67.

Rich JN, Zhang M, Datto MB, et al (1999). Transforming growth factor-beta-mediated p15(INK4B) induction and growth inhibition in astrocytes is SMAD3-dependent and a pathway prominently altered in human glioma cell lines. $J$ Biol Chem, 274, 35053-8.

Rifkin DB (2005). Latent transforming growth factor- $\beta$ (TGF- $\beta$ ) binding proteins: orchestrators of TGF- $\beta$ availability. J Biol Chem, 280, 7409-12.

Rodon L, Gonzalez-Junca A, Inda Mdel M, et al (2014). Active CREB1 promotes a malignant TGFbeta2 autocrine loop in glioblastoma. Cancer Discov, 4, 1230-41.

Roth P, Junker M, Tritschler I, et al (2010). GDF-15 contributes to proliferation and immune escape of malignant gliomas. Clin Cancer Res, 16, 3851-9.

Roth P, Silginer M, Goodman SL, et al (2013). Integrin control of the transforming growth factor- $\beta$ pathway in glioblastoma. Brain, 136, 564-76.

Sanchez-Elsner T, Botella LM, Velasco B, et al (2001). Synergistic cooperation between hypoxia and transforming growth factor-beta pathways on human vascular endothelial growth factor gene expression. J Biol Chem, 276, 38527-35.

Sánchez-Tilló E, Liu Y, de Barrios O, et al (2012). EMTactivating transcription factors in cancer: beyond EMT and tumor invasiveness. Cellular Molecular Life Sciences, 69 , 3429-56.

Santibanez JF, Quintanilla M, Bernabeu C (2011). TGF-beta/ TGF-beta receptor system and its role in physiological and pathological conditions. Clin Sci (Lond), 121, 233-51.

Savary K, Caglayan D, Caja L, et al (2013). Snail depletes the tumorigenic potential of glioblastoma. Oncogene, 32, 5409-20.

Schaffner F, Ray AM, Dontenwill M (2013). Integrin $\alpha 5 \beta 1$, the fibronectin receptor, as a pertinent therapeutic target in solid tumors. Cancers, 5, 27-47.

Scheel C, Weinberg RA (2012). Cancer stem cells and epithelialmesenchymal transition: concepts and molecular links. Semin Cancer Biol, 22, 396-403.

Seoane J, Le HV, Shen L, et al (2004). Integration of smad and forkhead pathways in the control of neuroepithelial and glioblastoma cell proliferation. Cell, 17, 211-23.

Shen L, Qu X, Ma Y, et al (2014). Tumor suppressor NDRG2 tips the balance of oncogenic TGF-[beta] via EMT inhibition in colorectal cancer. Oncogenesis, 3,86 .

Shnaper S, Desbaillets I, Brown DA, et al (2009). Elevated levels of MIC-1/GDF15 in the cerebrospinal fluid of patients are associated with glioblastoma and worse outcome. Int $J$ Cancer, 125, 2624-30.

Shull MM, Ormsby I, Kier AB, et al (1992). Targeted disruption of the mouse transforming growth factor-beta 1 gene results in multifocal inflammatory disease. Nature, 359, 693-9.

Siegel PM, Massague J (2003). Cytostatic and apoptotic actions of TGF-beta in homeostasis and cancer. Nat Rev Cancer, 3, 807-21.

Silginer M, Weller M, Ziegler U, et al (2014). Integrin inhibition promotes atypical anoikis in glioma cells. Cell Death Disease, 5, 1012 .

Singh SK, Hawkins C, Clarke ID, et al (2004). Identification of human brain tumour initiating cells. Nature, 432, 396-401.

Sottoriva A, Spiteri I, Piccirillo SG, et al (2013). Intratumor heterogeneity in human glioblastoma reflects cancer evolutionary dynamics. Proc Natl Acad Sci US A, 110, 4009-14.
Stupp R, Hegi ME, Gorlia T, et al (2014). Cilengitide combined with standard treatment for patients with newly diagnosed glioblastoma with methylated MGMT promoter (CENTRIC EORTC 26071-22072 study): a multicentre, randomised, open-label, phase 3 trial. Lancet Oncol, 15, 1100-8.

Sun G, Shi L, Li M, et al (2014a). Lefty inhibits glioma growth by suppressing nodal-activated smad and ERK1/2 pathways. $J$ Neurol Sci, 347, 137-42.

Sun J, Liu S-z, Lin Y, et al (2014b). TGF- $\beta$ promotes glioma cell growth via activating nodal expression through smad and ERK1/2 pathways. Biochem Biophys Res Communicat, 443, 1066-72.

Ten Dijke P, Arthur HM (2007). Extracellular control of TGFbeta signalling in vascular development and disease. Nat Rev Mol Cell Biol, 8, 857-69.

The Cancer Genome Atlas Research Network (2008). Comprehensive genomic characterization defines human glioblastoma genes and core pathways. Nature, 455, 1061-8.

Travis MA, Sheppard D (2014). TGF- $\beta$ activation and function in immunity. Ann Revi Immunol, 32, 51-82.

Tsuchida K, Nakatani M, Hitachi K, et al (2009). Activin signaling as an emerging target for therapeutic interventions. Cell Communicat Signal, 7, 15.

Tsukazaki T, Chiang TA, Davison AF, et al (1998). SARA, a FYVE Domain Protein that Recruits Smad2 to the TGF $\beta$ Receptor. Cell, 95, 779-91.

Uhl M, Aulwurm S, Wischhusen J, et al (2004). SD-208, a novel transforming growth factor beta receptor I kinase inhibitor, inhibits growth and invasiveness and enhances immunogenicity of murine and human glioma cells in vitro and in vivo. Cancer Res, 64, 7954-61.

Utsuki S, Oka H, Miyajima Y, et al (2012). Adult cerebellar glioblastoma cases have different characteristics from supratentorial glioblastoma. Brain Tumor Pathol, 29, 87-95.

van Tellingen O, Yetkin-Arik B, de Gooijer MC, et al (2015). Overcoming the blood-brain tumor barrier for effective glioblastoma treatment. Drug Resistance Updates.

Vergeli M, mazzanti B, Ballerini C, et al (1995). Transforming growth factor-beta 1 inhibits the proliferation of rat astrocytes induced by serum and growth factors. J Neurosci Res, 40, 127-33.

Verhaak RGW, Hoadley KA, Purdom E, et al (2010). An integrated genomic analysis identifies clinically relevant subtypes of glioblastoma characterized by abnormalities in PDGFRA, IDH1, EGFR and NF1. Cancer cell, 17, 98.

Wakefield LM, Hill CS (2013). Beyond TGFbeta: roles of other TGFbeta superfamily members in cancer. Nat Rev Cancer, 13, 328-41.

Wan YY, Flavell RA (2007). 'Yin-Yang' functions of transforming growth factor-beta and $\mathrm{T}$ regulatory cells in immune regulation. Immunol Rev, 220, 199-213.

Weiss A, Attisano L (2013). The TGFbeta superfamily signaling pathway. Wiley Interdisciplinary Reviews: Developmental Biol, 2, 47-63.

Wick W, Platten M, Weller M (2001). Glioma cell invasion: regulation of metalloproteinase activity by TGF-beta. $J$ Neurooncol, 53, 177-85.

Witsch E, Sela M, Yarden Y (2010). Roles for growth factors in cancer progression. Physiol (Bethesda), 25, 85-101.

Wrana JL (1998). TGF-beta receptors and signalling mechanisms. Miner Electrolyte Metab, 24, 120-30.

Wrana JL (2013). Signaling by the TGF $\beta$ superfamily. Cold Spring Harbor Perspect Biol, 5.

Xu J, Acharya S, Sahin O, et al (2015). 14-3-3 $\zeta$ Turns TGF- $\beta$ 's function from tumor suppressor to metastasis promoter in breast cancer by contextual changes of smad partners from p53 to Gli2. Cancer Cell, 27, 177-92. 
Overview of Transforming Growth Factor $\beta$ Superfamily Involvement in Glioblastoma Initiation and Progression

Yamada N, Kato M, Yamashita H, et al (1995). Enhanced expression of transforming growth factor-beta and its type-I and type-II receptors in human glioblastoma. Int J Cancer, 62, 386-92.

Yan K, Wu Q, Yan DH, et al (2014). Glioma cancer stem cells secrete Gremlin1 to promote their maintenance within the tumor hierarchy. Genes Develop, 28, 1085-100.

Yang X, Letterio JJ, Lechleider RJ, et al (1999). Targeted disruption of SMAD3 results in impaired mucosal immunity and diminished T cell responsiveness to TGF-beta. Embo J, 18, 1280-91.

Ye S, Li P, Tong C, et al (2013). Embryonic stem cell self-renewal pathways converge on the transcription factor Tfcp211. EMBO Journal, 32, 2548-60.

Ye XZ, Xu SL, Xin YH, et al (2012). Tumor-associated microglia/ macrophages enhance the invasion of glioma stem-like cells via TGF-beta1 signaling pathway. J Immunol, 189, 444-53.

Zagzag D, Salnikow K, Chiriboga L, et al (2005). Downregulation of major histocompatibility complex antigens in invading glioma cells: stealth invasion of the brain. Lab Invest, $\mathbf{8 5}$, 328-41.

Zhang DF, Li XG, Su LJ, et al (2010). Expression of activin A and follistatin in glioblastoma and their effects on U87 in vitro. J Int Med Res, 38, 1343-53.

Zhang L, Sato E, Amagasaki K, et al (2006). Participation of an abnormality in the transforming growth factor-beta signaling pathway in resistance of malignant glioma cells to growth inhibition induced by that factor. $J$ Neurosurg, 105, 119-28.

Zong H, Parada LF, Baker SJ (2015). Cell of origin for malignant gliomas and its implication in therapeutic development. Cold Spring Harb Perspect Biol. 